\title{
Charcot-Marie-Tooth disease and related hereditary polyneuropathies: Molecular diagnostics determine aspects of medical management
}

\author{
Kinga Szigeti, $M D^{1}$, Carlos A. Garcia, $M D^{3}$, and James R. Lupski, $M D, P h D^{1,2}$
}

\begin{abstract}
Purpose: An evidence-based approach was used to determine the frequency distribution of genes contributing to the Charcot-Marie-Tooth (CMT) disease phenotype. Methods: We performed a combined analysis of 11 populationbased studies from various ethnic backgrounds to generate an evidence-based testing scheme. To estimate the relative frequencies of the responsible genes for which population-based studies are not available, we used our cohort of clinically classified patients with CMT and related neuropathies collected before the availability of genetic testing. Results: Similar mutation frequencies were detected in the various studies, revealing a uniform distribution of pathogenic mutations. In CMT1 70\% of patients harbor the CMT1A duplication, followed by GJB1 mutations at $8.8 \%$. MPZ and PMP22 mutations are less common, identified on average in $2.9 \%$ and $1.5 \%$ of patients, respectively. Other genes not tested in population-based studies contribute to less than $1 \%$ of disease individually. In CMT2 MFN2 mutations are the most common, although population-based studies are not yet available. Conclusion: CMT represents a heterogeneous group of disorders at the molecular level. Nevertheless, testing for the CMT1A duplication (i.e., duplication of PMP22) alone yields an accurate molecular diagnosis in approximately half of all patients. If one further specifies the clinical type (demyelinating vs. axonal), the yield of detecting a molecular defect increases to $75 \%$ to $80 \%$ in the demyelinating or CMT1 group with a screening test that evaluates for CMT1A duplication/hereditary neuropathy with liability to pressure palsies deletion and GJB1 point mutations.
\end{abstract} Genet Med 2006:8(2):86-92.

Key Words: population study, evidence-based medicine, DNA diagnosis, duplication

Case vignette: A 35-year-old man presents with a 5-year history of numbness in his feet and occasional tripping. Family history reveals that he has an 8-year-old daughter. He and his wife lost a 5-year-old daughter 1 year ago, who developed acute lymphoblastic leukemia and became paralyzed after the first dose of chemotherapy. His wife is expecting another child.

Signs and symptoms of peripheral neuropathy are often identified during office visits to both the neurologist and generalist physician. When neuropathy presents in the context of a systemic illness, such as diabetes or uremia, the acquired nature is easily recognized. However, in the absence of overt systemic illness, the ability to distinguish acquired from inherited

\footnotetext{
From the Departments of ${ }^{1}$ Molecular and Human Genetics and ${ }^{2}$ Pediatrics, Baylor College of Medicine, and ${ }^{2}$ Texas Children Hospital, Houston, Texas; ${ }^{3}$ Departments of Neurology and Pathology, Tulane University Health Sciences Center, New Orleans, Louisiana.

James R. Lupski, MD, PhD, Baylor College of Medicine, One Baylor Plaza, Rm604B, Houston, TX 77030.

Submitted for publication July 19, 2005.

Accepted for publication December 1, 2005.

Disclosure: J.R.L. is a co-inventor of a patented molecular diagnostic test for CMT (US Patents 5,306,616; 5,599,920; 5,780,223) and a consultant for Athena Diagnostics.

DOI: 10.1097/01.gim.0000200160.29385.73
}

neuropathies on clinical grounds is often challenging, particularly in sporadic disease.

Charcot-Marie-Tooth (CMT) and related peripheral neuropathies represent a heterogeneous group of hereditary disorders of the peripheral nervous system with an estimated frequency of 1 in 2500 individuals. ${ }^{1}$ CMT is characterized by slow and progressive weakness of the legs followed, in some cases, by hand involvement. On the basis of motor nerve conduction velocities (NCVs), two major types can be distinguished: the demyelinating form (CMT1), which is characterized by symmetrically slowed NCV (usually $<38 \mathrm{~m} / \mathrm{sec}$; normal is $>45$ $\mathrm{m} / \mathrm{sec}$ ), and the axonal form (CMT2), associated with normal or subnormal NCV and reduced compound muscle action potential. Both the demyelinating and the axonal forms can be inherited as an autosomal dominant, autosomal recessive, or $\mathrm{X}$-linked trait, but often present as sporadic neuropathy.

During the last decade an enormous amount of information regarding peripheral nerve function and dysfunction has been obtained through the identification of genes responsible for disease in patients manifesting inherited peripheral neuropathies. ${ }^{2,3}$ Some of these genes/mutations contribute to a significant fraction of inherited peripheral neuropathy cases. Thus, molecular analyses can play a substantial role in establishing a precise and accurate etiologic diagnosis, whereas other genes may be in- 
volved in only a minority of patients. A molecular diagnosis not only establishes a secure diagnosis but also enables accurate recurrence risk estimates, provides potential prognostic information, identifies those possibly at risk for idiosyncratic drug reactions, and allows the possibilities for prenatal diagnosis.

Molecular testing for inherited neuropathy is clinically available. For the CMT1A duplication/hereditary neuropathy with liability to pressure palsies (HNPP) deletion, quantitative and qualitative approaches have been implemented. Typically, diagnostic laboratories in the United States use fluorescence in situ hybridization or pulsed-field gel electrophoresis, both of which are based on the detection of the presence or absence of a signal or band. Both methods have a $100 \%$ analytic sensitivity and specificity, and detect both the duplication and the reciprocal deletion that is responsible for a distinct neuropathy known as HNPP. More recently, multiplex ligation-dependent probe amplification ${ }^{4}$ and array comparative genome hybridization ${ }^{5}$ have also been used in the United States. In Europe, multiple additional molecular methods $s^{6,7}$ have been used to detect the CMT1A duplication and HNPP deletion, several of which quantitate PMP22 gene copy number as a basis for the assay. The detection of point mutations in any of the CMT-causing genes requires sequencing of the appropriate genes, and lack of clustering of mutations necessitates screening the entire coding region. Direct sequencing also has a 100\% analytic sensitivity and specificity.

Mutations or copy number alterations of more than 24 genes have been shown to cause CMT and related peripheral neuropathies generating a complicated molecular classification and making it difficult to apply the vast amount of information in clinical practice (http://molgen-www.uia.ac.be/ CMTMutations). Testing for disease-associated mutations in each of the potential causative genes is neither practical nor cost-effective. We performed a combined analysis of 11 population-based studies to identify the clinically relevant genes and mutations and to generate an evidenced-based testing scheme. We also used our own cohort of patients collected before the availability of genetic testing to estimate the relative frequency of the responsible genes for which population-based studies are not available. Our evaluations suggest a systematic approach to molecular testing in patients with hereditary neuropathy. We propose a screening molecular test to capture the most frequently occurring pathogenic alleles and at the same time provide information that impinges directly on clinical management.

\section{METHODS}

To determine the frequency of the mutations in the various genes underlying CMT and related peripheral neuropathy we performed a PubMed search with the headings charcot and mutation (734 hits), and after review of the abstracts we identified 11 population-based studies ${ }^{8-18}$ reporting on genetic testing and frequencies of genes involved in CMT and related peripheral neuropathy.
A cohort of 153 unrelated patients with CMT collected before the clinically availability of genetic testing (i.e., before circa 1993) was analyzed to estimate the contribution of the genes not reported in the population-based studies. All patients referred to this study by their primary physician or neurologist received appropriate counseling and gave informed consent approved by the institutional review board of Baylor College of Medicine. The clinical diagnosis was based on clinical examination, electrophysiologic studies, and, in a few cases, nerve biopsy given by a neurologist (C.A.G. in most cases). CMT1 was defined as symmetrically decreased $\mathrm{NCV}(<38 \mathrm{~m} / \mathrm{sec}$ in the lower extremities), and CMT2 was defined as normal or subnormal NCV and reduced compound muscle action potential.

Preliminary mutation studies in this cohort for selected CMT-associated genes have been reported. ${ }^{12}$ The cohort was screened for the CMT1A duplication, the HNPP deletion, and the point mutations in GJB1, MPZ, PMP22, EGR2, PRX, NEFL, SOX10, SIMPLE, GDAP1, LMNA, TDP1, and MTMR2. The CMT1A duplication/HNPP deletion testing (which tests for the duplication or deletion of PMP22) was performed by pulsed-field gel electrophoresis, whereas point mutations were detected by denaturing high-performance liquid chromatography followed by direct sequencing of the abnormal amplicons by protocols published earlier or by direct sequencing of all coding exons. ${ }^{19}$ The primers and conditions are available on the laboratory's website (http://www.imgen.bcm.tmc.edu/ molgen/lupski/).

\section{RESULTS}

We determined the frequency contribution of individual genes and mutations from a combined analysis of 11 population-based studies including patients from various ethnic backgrounds. ${ }^{8-18}$ These studies reported results on five genes and genomic rearrangements: PMP22 duplication/deletion (i.e., the CMT1A duplication/HNPP deletion), GJB1, MPZ, and PMP22 point mutations. We analyzed mutation frequency in the total population with CMT in whom the specific type was not determined (Table 1), in patients in whom the type 1 or the demyelinating form of CMT (i.e., CMT1) was specified (Table 2), and in all phenotypic subgroups (Table 3 ).

The most common mutation identified was the CMT1A duplication. This single mutation was found, on average, in $43 \%$ of patients with CMT (Table 1), with that frequency increasing to $70 \%$ when the diagnosis was further specified as CMT1 using objective clinical testing (Table 2). For CMT1 the next most commonly mutated gene was GJB1, encoding Cx32 responsible for the X-linked form of CMT, accounting on average for $8.8 \%$ of patients with CMT1 (Table 2), whereas PMP22 and $M P Z$ mutations accounted for $1 \%$ to $3 \%$ (Table 2).

The distribution of genes/mutations causing CMT was evaluated in our cohort of patients with CMT and related inherited neuropathy to assess the frequency contributions of individual genes not evaluated in the population-based studies. ${ }^{12}$ This cohort contains patients referred before the clinical diagnostic availability of molecular testing; thus, the frequencies may be 
Table 1

Mutation frequencies for Charcot-Marie-Tooth disease and related neuropathies in seven population studies

\begin{tabular}{|c|c|c|c|c|c|c|}
\hline Population & $\begin{array}{c}\text { Cohort } \\
\text { (No. of patients) }\end{array}$ & $\begin{array}{l}\text { CMT1A duplication } \\
(\%)\end{array}$ & $\begin{array}{l}\text { HNPP deletion } \\
(\%)\end{array}$ & $\begin{array}{c}\text { PMP22 mutation } \\
(\%)\end{array}$ & $\begin{array}{c}\text { Cx32 mutation } \\
(\%)\end{array}$ & $\begin{array}{c}M P Z \text { mutation } \\
(\%)\end{array}$ \\
\hline American ${ }^{10,12}$ & 75 & 56 & ND & 3.9 & 7.2 & 3.3 \\
\hline Spanish $^{13}$ & 52 & Excluded & Excluded & $0.8^{a}$ & $7.7^{a}$ & $3.8^{a}$ \\
\hline Belgian $^{14}$ & 443 & 24.6 & 10.6 & 2.7 & 5.4 & 0.7 \\
\hline Finnish $^{9}$ & 157 & 40.7 & 26.1 & ND & 7.6 & ND \\
\hline European $^{11}$ & 975 & 59.4 & 13.4 & ND & ND & ND \\
\hline Russian $^{16}$ & 174 & 33.9 & ND & 1.1 & 6.8 & 3.4 \\
\hline Korean $^{18}$ & 57 & 26 & ND & 1.7 & 5.3 & 5.3 \\
\hline Average & & $43 \%$ & $11 \%$ & $2.5 \%$ & $6.9 \%$ & $3.5 \%$ \\
\hline
\end{tabular}

${ }^{a}$ Extrapolated total number and mutation frequencies recalculated for the total number. For the estimation of the total number we calculated with the average frequencies for CMT1A duplication and HNPP deletion derived from the other studies.

ND, not determined; CMT1A, Charcot-Marie-Tooth disease type 1A; HNPP, hereditary neuropathy with liability to pressure palsies.

Table 2

Mutation frequencies for Charcot-Marie-Tooth disease type 1 in seven population studies

\begin{tabular}{|c|c|c|c|c|c|}
\hline Population & $\begin{array}{c}\text { Cohort } \\
\text { (No. of patients) }\end{array}$ & $\begin{array}{l}\text { CMT1A duplication } \\
(\%)\end{array}$ & $\begin{array}{c}\text { PMP22 mutation } \\
(\%)\end{array}$ & $\begin{array}{c}C \times 32 \text { mutation } \\
(\%)\end{array}$ & $\begin{array}{c}M P Z \text { mutation } \\
(\%)\end{array}$ \\
\hline American $^{10,12}$ & 63 & 68 & ND & ND & ND \\
\hline Slovene $e^{15}$ & 71 & 81 & ND & ND & ND \\
\hline European $^{11}$ & 819 & 70.7 & ND & ND & ND \\
\hline Australian ${ }^{8}$ & 224 & 61 & 1.3 & 12 & 3.1 \\
\hline Russian ${ }^{16}$ & 108 & 53.7 & 1.9 & 7.4 & 3.4 \\
\hline Italian $^{17}$ & 172 & 57.6 & 1.2 & 6.9 & 2.3 \\
\hline Korean $^{18}$ & 28 & 54 & ND & ND & ND \\
\hline Average & & $70 \%$ & $1.5 \%$ & $8.8 \%$ & $2.9 \%$ \\
\hline
\end{tabular}

CMT1A, Charcot-Marie-Tooth disease type 1A; ND, not determined.

Table 3

Mutation frequency in clinically classified neuropathy phenotypic subgroups (literature data ${ }^{8-18}$ )

\begin{tabular}{|c|c|c|c|c|c|}
\hline & $\begin{array}{c}\text { PMP22 } \\
\text { duplication }\end{array}$ & $\begin{array}{l}P M P 22 \\
\text { deletion }\end{array}$ & PMP22 & $\begin{array}{c}\text { Mutation } \\
C \times 32\end{array}$ & $M P Z$ \\
\hline CMT1 & $54-81$ & ND & $0-2$ & 5-19 & $2-7$ \\
\hline HNPP & ND & $84-100$ & $0-20$ & ND & ND \\
\hline CMT2 & 0 & 0 & 0 & $3-57$ & 0 \\
\hline DSN & 0 & 0 & $14-50$ & 0 & $14-25$ \\
\hline
\end{tabular}

CMT1, Charcot-Marie-Tooth disease type 1A; HNPP, hereditary neuropathy with liability to pressure palsies; CMT2, Charcot-Marie-Tooth disease type 2; CMT, Charcot-Marie-Tooth disease, type 1 or 2 not specified; DSN, DejerineSotas neuropathy; ND, not determined.

less prone to bias from selective testing. The frequency contributions for pathogenic mutations are shown in Table 4. In approximately $70 \%$ of patients a molecular diagnosis could be achieved. Remarkably, the CMT1A duplication accounts for approximately $50 \%$ of neuropathy and $70 \%$ (79/113) of demyelinating neuropathy; this latter value is consistent with previous population-based studies of $68 \%{ }^{10}$ and $70 \% .^{11}$ As also found in other studies, in CMT GJB1 (Cx32) mutations were the next most frequently observed (7\%) followed by PMP22 and $M P Z$ point mutations (3\%-4\%). Mutations in other genes (e.g., EGR2, PRX, MTMR2, and NEFL) were identified less frequently in this mixed group of patients with CMT or related neuropathy never accounting for more than $1 \%$ of mutations in this limited patient sample.

Molecular diagnostic screening in clinical diagnostic laboratories, with a less clinically defined cohort, also revealed the CMT1A duplication as the most frequently observed mutation (data not shown) in patients with hereditary neuropathy. The CMT1A duplication was identified more frequently than the HNPP deletion, and mutations in GJB1 were the next most frequently found.

\section{DISCUSSION}

\section{Prioritizing molecular testing}

Hereditary polyneuropathy represents $42 \%$ of 205 cases of undiagnosed neuropathy ${ }^{20}$ and $30 \%$ of 402 consecutive patients with polyneuropathy ${ }^{21}$ in two separate neuropathy specialty clinics. Thus, hereditary polyneuropathy was recognized to account 
Table 4

Mutation frequencies in 153 unrelated, clinically classified cases of Charcot Marie-Tooth disease or related neuropathy before commercial testing

\begin{tabular}{|c|c|c|c|c|c|c|c|c|c|c|}
\hline Type & $\operatorname{Dup}(17 p)$ & $C \times 32$ & $M P Z$ & PMP22 & EGR2 & $P R X$ & MTMR2 & NEFL & Unknown & Total \\
\hline CMT1 & 79 & 8 & 5 & 3 & 1 & & & & 11 & 107 \\
\hline HNPP & & & & & & & & & 1 & 1 \\
\hline DSN & & & & 2 & & 1 & & & 1 & 4 \\
\hline $\mathrm{CHN}$ & & & & & & & & & 1 & 1 \\
\hline CMT2 & & 3 & & & & & & 1 & 2 & 6 \\
\hline Other & & & & 1 & & & & & 33 & 34 \\
\hline Total & 79 & 11 & 5 & 6 & 1 & 1 & 0 & 1 & 49 & 153 \\
\hline$\%$ & 51.6 & 7.2 & 3.3 & 3.9 & 0.7 & 0.7 & 0.0 & 0.7 & 32 & 100 \\
\hline
\end{tabular}

CMT1, Charcot Marie-Tooth disease type 1A; HNPP, hereditary neuropathy with liability to pressure palsies; DSN, Dejerine-Sotas neuropathy; CHN, congenital hypomyelinating neuropathy; CMT2, Charcot-Marie-Tooth disease type 2.

for a significant fraction of all neuropathy even in an era before the clinical availability of molecular diagnostics. Because some of the cryptogenic, drug-induced, and multifocal motor neuropathies may have a genetic cause, and genetic neuropathies can be caused by de novo mutations, a genetic cause may underlie between $30 \%$ and $50 \%$ of polyneuropathies. ${ }^{21}$

The 11 population-based studies from various ethnic backgrounds ${ }^{8-18}$ reported molecular diagnostic results on five genes and genomic rearrangements: PMP22 duplication/deletion, GJB1, MPZ, and PMP22 point mutations. Despite different cohorts, recruitments, and testing protocols, similar mutation frequencies were detected in the various population-based studies, revealing a uniform distribution of pathogenic mutations. The application of a simple clinical classification, demyelinating (CMT1) versus axonal (CMT2) neuropathy (Tables 1 and 2), and consideration of the inheritance pattern markedly improved the diagnostic yield.

Molecular testing in clinical diagnostic laboratories confirmed the relative frequencies of the various genes harboring pathogenic mutations; however, the pattern of ordering molecular testing is sometimes skewed toward panel testing that simultaneously examines for multiple genetic causes (data not shown). This may reflect that practicing physicians have difficulty in prioritizing individual tests with the overwhelming amount of molecular genetic information available. Furthermore, the positive yield of testing was decreased (20\%-25\% positive for CMT1A duplication; data not shown), likely reflecting the less stringent diagnostic criteria applied in a clinical setting using molecular testing to establish one of many possibilities in a differential diagnosis.

Duplication of a chromosomal segment harboring PMP22 (i.e., the CMT1A duplication) ${ }^{22}$ on average represents $43 \%$ of the total CMT cases, whereas the yield of duplication detection increases to 70\% in CMT1 (Tables 1, 2, and 4). Identification of the CMT1A duplication establishes the neuropathy as an autosomal dominant trait in the patient's family. This group of patients are also the potential beneficiaries of the novel therapeutic interventions and should be identified to enroll them in controlled clinical trials. Of the previously cited 402 patients with polyneuropathy, ${ }^{21}$ we can conservatively predict that approximately 52 patients, $43 \%$ of the 121 inherited neuropathy cases, will have duplication of PMP22 or the CMT1A duplication. Because the CMT1A duplication is the most common mutation causing neuropathy, it is likely that drug-toxicity studies will address this population initially, just like data of idiosyncratic vincristine toxicity have emerged from patients with this mutation. ${ }^{23,24}$

The deletion of the same chromosomal segment results in HNPP. ${ }^{25,26}$ HNPP can mimic multifocal neuropathy, a frequently inflammatory disorder that requires immunosuppressant therapy. The individuals with HNPP among this group of patients need to be identified to do no harm. Although detection of deletion has a low yield in the population with CMT, the clinical picture is distinctive and the deletion is specific for HNPP; thus, testing of this phenotype identifies deletion mutations in more than $84 \%$ of patients (Table 3 ). Of 52 consecutive patients with multifocal neuropathy, $47 \%$ had $P M P 22$ deletion, the knowledge of which completely changes management, permitting avoidance of immunosuppressant therapy and initiation of measures to prevent pressure injury. ${ }^{27}$

After the CMT1A duplication and HNPP deletion, GJB1 mutations are the next most common culprits in inherited neuropathy (Tables 1-4). The phenotype can be more intermediate, with features of demyelination and axonal loss especially in females with this X-linked dominant trait. A dominant inheritance pattern and lack of male-to-male transmission indicate this gene on the X chromosome. Identification of a GJB1 mutation determines an X-linked dominant inheritance pattern, enabling both genetic counseling and accurate estimation of recurrence risk.

The population-based studies suggest that in patients with the demyelinating phenotype, $M P Z$ and $P M P 22$ mutations are the next most common after PMP22 duplication and GJB1 mutations (Table 2). Molecular diagnostic studies to examine gene/mutation frequency distributions in large cohorts of patients with CMT2 are in their infancy. Recent data, although not population based, suggest that MFN2 mutations may be one of the most common causes of axonal CMT or CMT2. ${ }^{28,29}$ 
MFN2 mutations may account for up to $20 \%$ of CMT2,30,31 but limited studies and no population data are available thus far.

Other genes are less commonly involved in the population with CMT; however, unique clinical features may indicate a specific gene. External ophthalmoplegia and respiratory compromise are observed with mutations in EGR2, whereas profound sensory loss implicates $P R X$ mutations. The sensitivity or specificity of these unique features is not currently available. Nonetheless, establishing a molecular diagnosis makes genetic counseling more accurate and provides an opportunity to study the natural history of the diseases in a molecularly homogeneous group. Such molecular stratification may enable better prognostication. Moreover, as further therapeutic modalities emerge, it is likely that they will be specific to the gene involved and the type of mutation identified within that gene. ${ }^{32}$

Because of genetic heterogeneity, the diagnostic sensitivity of molecular testing does not approximate the high analytic sensitivities of the molecular methods themselves. Multiple analyses need to be performed to reach a high diagnostic sensitivity (Fig. 1), but a stepwise approach based on the frequencies will result in substantial savings of medical resources. The extent of genetic testing should be guided by specific questions and individualized accordingly.

\section{Screening molecular diagnostics in adults and children with suspected inherited polyneuropathy}

The focus and the reasons for obtaining a molecular diagnosis are different in the adult and pediatric populations. In adults with the CMT phenotype, PMP22 duplication and GJB1 mutation analyses establish the molecular diagnosis in approximately half of all patients (Table 1). The combination of PMP22 duplication and GJB1 mutation testing identifies the candidates for clinical trials, and if these trials reveal novel treatment options, ultimately identifies those who may benefit from treatment. It also identifies families whose members are potentially at risk for idiosyncratic drug reactions and determines the inheritance pattern establishing a secure diagnosis and grounds for accurate genetic counseling and prenatal diagnosis. If patients with the demyelinating form are tested as a group, the diagnostic yield increases to $75 \%$ to $80 \%$ by initially performing just PMP22 duplication and GJB1 mutation analyses as a screening test (Table 5, Fig. 1).

In the pediatric population the aim of testing has a different emphasis. The parents frequently have a child with severe weakness and normal intellect. The parents' major concerns are prognosis and recurrence risk given their interest in the possibilities of treatment by participation in clinical trials and their desire to have additional children. The answers to their questions will depend on an accurate molecular diagnosis. Thus in children after testing for the common causes of peripheral neuropathy, PMP22 duplication and GJB1 mutations, the physician should proceed to panel testing for all the genes. As of yet we are still learning, and what we have ascertained to date is that the clinical phenotypes of the various genes overlap. Sometimes certain unique clinical features indicate a specific etiologic gene, such as marked sensory loss implicates periaxin mutations or respiratory compromise and external ophthalmoplegia suggest EGR2 mutations; other times the clinical feature can be observed with many different gene mutations, such as hearing loss. Occasionally the inheritance pattern helps guide the sequence of genetic testing, for example, autosomal recessive inheritance may implicate $P R X$ or GDAP1 mutations. For some of the genetic alterations prenatal diagnosis is available (www.geneclinics.org).

\section{New mutations}

The high frequency of de novo mutations in duplication/ deletion $(37 \%-90 \%)^{11,33}$ illustrates that genetic disease is often

\section{Percent yield}

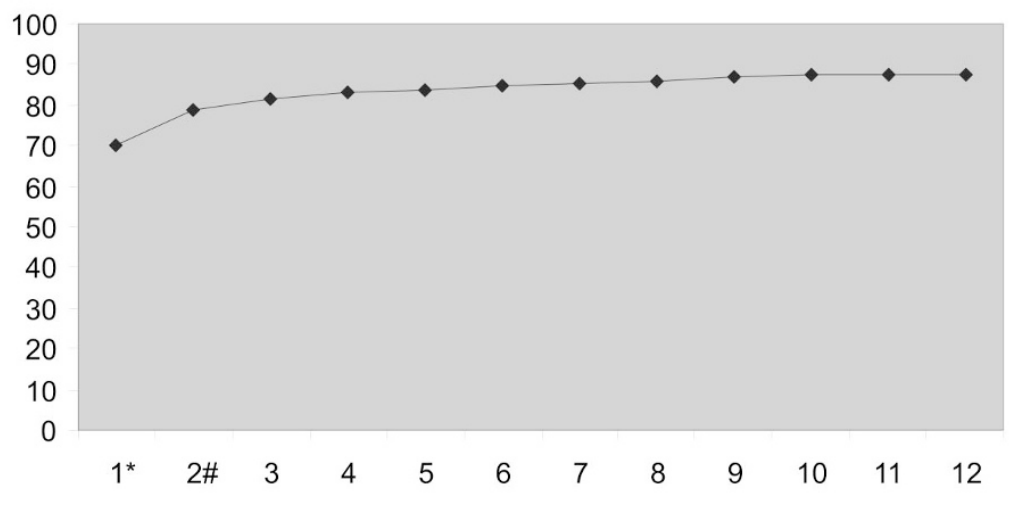

Number of genes tested

\section{* PMP22 duplication \\ \# GJB1 point mutation}

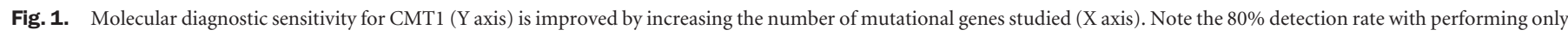
two tests. 
Table 5

Molecular diagnostic screening in patients with suspected inherited polyneuropathy

\begin{tabular}{llc}
\hline Diagnosis & \multicolumn{1}{c}{ Screening test } & Establish molecular diagnosis \\
\hline CMT & CMT1A dup/HNPP del & $45 \%-50 \%^{a}$ \\
& GJB1 point mutation & \\
CMT1 & CMT1A dup/HNPP del & $75 \%-80 \%$ \\
& GJB1 point mutation & \\
CMT2 & MFN2, GJB1, MPZ & $20 \%-25 \%^{b}$ \\
\hline
\end{tabular}

${ }^{a}$ Evidence based from population studies.

${ }^{b}$ Extrapolated.

CMT, Charcot Marie-Tooth disease; CMT1, Charcot-Marie-Tooth disease type 1 or demyelinating; CMT2, Charcot-Marie-Tooth disease type 2 or axonal.

sporadic in presentation. Thus, the lack of a family history does not preclude molecular testing. In our cohort point mutations often occurred de novo, in 6 of 16 patients (38\%). ${ }^{12}$ The frequently occurring de novo event for both rearrangements and point mutations necessitates that one has an index of suspicion for genetic disease even in the absence of a family history. In fact, in a patient presenting with chronic polyneuropathy in the absence of other signs and symptoms, after the most common systemic and treatable causes, such as diabetes, uremia, and nutritional deficiency, genetic causes are more common than autoimmune or paraneoplastic neuropathy.

\section{Areas of uncertainty}

Molecular testing occasionally identifies new sequence variations of unknown pathogenic significance. In these cases further studies (segregation analysis, functional assay) are required to establish pathogenicity, which are performed in research laboratories. These results provide useful information, but have to be interpreted and relayed to the patient as research data. However, the ambiguous results affect only a small number of patients, not more frequently than borderline autoimmune test results in immune-mediated neuropathy.

\section{CONCLUSIONS}

Hereditary polyneuropathy is common, and powerful diagnostic tests are clinically available. Recently, two potential small molecule therapeutic approaches ${ }^{34,35}$ proved effective in the treatment of animal models for a specific molecular form of CMT neuropathy, which is caused by PMP22 duplication. These observations will initiate clinical trials and have the potential to ultimately lead to molecular-based therapy in patients with the CMT1A duplication. This could result in a completely different clinical management focus: from the possibility of molecular diagnosis to the failure to treat potential beneficiaries. Published reports on vincristine toxicity in susceptible presymptomatic groups of patients ${ }^{23,24,36,37}$ underlie the necessity of awareness of molecular diagnostic possibilities. Furthermore, a molecular diagnosis helps avoid immunosuppressant treatment in HNPP, complying with the oath of "do no harm."
Although inherited peripheral neuropathy represents a heterogeneous group of disorders at the molecular level, testing for a few genes/mutations yields an accurate molecular diagnosis in more than half of patients. If we apply a simple clinical classification CMT1 (demyelinating) versus CMT2 (axonal), the yield of detecting a molecular defect increases to $75 \%$ to $80 \%$ in the demyelinating group by testing just for the CMT1A duplication and GJB1 mutations (Table 5, Fig. 1).

In the case vignette, the patient has CMT by physical examination. His daughter acquired acute lymphoblastic leukemia when she was presymptomatic for CMT at age 5 years. She had an idiosyncratic drug reaction to vincristine: a severe generalized acute polyneuropathy and respiratory failure, which is a known complication of vincristine treatment in patients with CMT. The patient's wife is pregnant, and they want to know the recurrence risk of CMT for the baby. Molecular genetic testing for PMP22 duplication and GJB1 mutation in the father has on average $50 \%$ (if type of CMT not further specified) to $79 \%$ (if CMT1) yield of concluding an accurate molecular diagnosis, which could answer their questions.

\section{AKNOWLEDGMENTS}

Carlos A. Garcia, MD, died on June 28, 2005. This article is dedicated to him and his lifelong interest in, and caring for, patients with CMT. We thank Sau W. Cheung, PhD, and Pawel Stankiewicz, MD, PhD, from the Kleberg Cytogenetics Laboratory, Baylor College of Medicine, and Uma Ananth, $\mathrm{PhD}$, and Amar Kamath from Athena Diagnostics for sharing their unpublished data on clinical testing.

\section{References}

1. Lupski JR, Garcia A. Charcot-Marie-Tooth peripheral neuropathies and related disorders. In: The Metabolic and Molecular Bases of Inherited Diseases. Vol. 4, Chapter 227. Eighth ed. Scriver CR, Beaudet AL, Sly WS et al (Eds.). New York: McGraw-Hill 2001:5759-5788.

2. Saifi GM, Szigeti K, Snipes GJ, Garcia CA et al. Molecular mechanisms, diagnosis, and rational approaches to management of and therapy for Charcot-Marie-Tooth disease and related peripheral neuropathies. J Investig Med 2003;51:261-283.

3. Suter U, Scherer SS. Disease mechanisms in inherited neuropathies. Nat Rev Neurosci 2003;4:714-726.

4. Schouten JP, McElgunn CJ, Waaijer R, Zwijnenburg D et al. Relative quantification of 40 nucleic acid sequences by multiplex ligation-dependent probe amplification. Nucleic Acids Res 2002;30:e57.

5. Cheung SW, Shaw CA, Yu W, Li J et al . Development and validation of a CGH microarray for clinical cytogenetic diagnosis. Genet Med 2005;7:422-432.

6. Patitucci A, Muglia M, Magariello A, Gabriele AL et al. Comparison of different techniques for detecting 17p12 duplication in CMT1A. Neuromuscul Disord 2005; 15:488-492

7. Thiel CT, Kraus C, Rauch A, Ekici AB et al. A new quantitative PCR multiplex assay for rapid analysis of chromosome 17p11.2-12 duplications and deletions leading to HMSN/HNPP. Eur J Hum Genet 2003;11:170-178.

8. Nicholson GA. Mutation testing in Charcot-Marie-Tooth neuropathy. Ann N Y Acad Sci 1999;883:383-388.

9. Silander K, Meretoja P, Juvonen V, Ignatius J et al. Spectrum of mutations in Finnish patients with Charcot-Marie-Tooth disease and related neuropathies. Hum Mutat 1998;12:59-68.

10. Wise CA, Garcia CA, Davis SN, Heju Z et al. Molecular analyses of unrelated Charcot-Marie-Tooth (CMT) disease patients suggest a high frequency of the CMTIA duplication. Am J Hum Genet 1993;53:853-863.

11. Nelis E, Van Broeckhoven C, De Jonghe P, Lofgren A et al. Estimation of the mutation frequencies in Charcot-Marie-Tooth disease type 1 and hereditary neuropathy with liability to pressure palsies: a European collaborative study. Eur J Hum Genet 1996;4:25-33. 


\section{Szigeti et al.}

12. Boerkoel CF, Takashima H, Garcia CA, Olney RK et al. Charcot-Marie-Tooth disease and related neuropathies: mutation distribution and genotype-phenotype correlation. Ann Neurol 2002;51:190-201.

13. Bort $\mathrm{S}$, Nelis E, Timmerman V, Sevilla $\mathrm{T}$ et al. Mutational analysis of the $M P Z$ $P M P 22$ and $C x 32$ genes in patients of Spanish ancestry with Charcot-Marie-Tooth disease and hereditary neuropathy with liability to pressure palsies. Hum Genet 1997;99:746-754.

14. Janssen EA, Kemp S, Hensels GW, Sie OG et al. Connexin32 gene mutations in X-linked dominant Charcot-Marie-Tooth disease (CMTX1). Hum Genet 1997;99: 501-505.

15. Leonardis L, Zidar J, Ekici A, Peterlin B et al. Autosomal dominant Charcot-MarieTooth disease type $1 \mathrm{~A}$ and hereditary neuropathy with liability to pressure palsies: detection of the recombination in Slovene patients and exclusion of the potentially recessive Thr118Met PMP22 point mutation. Int J Mol Med 1998;1:495-501.

16. Mersiyanova IV, Ismailov SM, Polyakov AV, Dadali EL et al. Screening for mutations in the peripheral myelin genes PMP22, MPZ and Cx32 (GJB1) in Russian Charcot-Marie-Tooth neuropathy patients. Hum Mutat 2000;15:340-347.

17. Mostacciuolo ML, Righetti E, Zortea M, Bosello V et al. Charcot-Marie-Tooth disease type I and related demyelinating neuropathies: mutation analysis in a large cohort of Italian families. Hum Mutat 2001;18:32-41.

18. Choi BO, Lee MS, Shin SH, Hwang JH et al. Mutational analysis of PMP22, MPZ, GJB1, EGR2 and NEFL in Korean Charcot-Marie-Tooth neuropathy patients. Hum Mutat 2004;24:185-186.

19. Takashima H, Boerkoel CF, Lupski JR. Screening for mutations in a genetically heterogeneous disorder: DHPLC versus DNA sequence for mutation detection in multiple genes causing Charcot-Marie-Tooth neuropathy. Genet Med 2001;3:335342.

20. Dyck PJ, Oviatt KF, Lambert EH. Intensive evaluation of referred unclassified neuropathies yields improved diagnosis. Ann Neurol 1981;10:222-226.

21. Barohn RJ. Approach to peripheral neuropathy and neuronopathy. Semin Neurol 1998;18:7-18.

22. Lupski JR, de Oca-Luna, RM, Slaugenhaupt S, Pentao L et al. DNA duplication associated with Charcot-Marie-Tooth disease type 1A. Cell 1991;66:219-232.

23. Chauvenet AR, Shashi V, Selsky C, Morgan E et al. Vincristine-induced neuropathy as the initial presentation of Charcot-Marie-Tooth disease in acute lymphoblastic leukemia: a Pediatric Oncology Group study. J Pediatr Hematol Oncol 2003;25:316-320.

24. Graf WD, Chance PF, Lensch MW, Eng LJ et al. Severe vincristine neuropathy in Charcot-Marie-Tooth disease type 1A. Cancer 1996;77:1356-1362.
25. Chance PF, Alderson MK, Leppig KA, Lensch MW et al. DNA deletion associated with hereditary neuropathy with liability to pressure palsies. Cell 1993;72:143-151.

26. Lupski JR, Chance PF, Garcia CA. Inherited primary peripheral neuropathies. Molecular genetics and clinical implications of CMT1A and HNPP. J Am Med Assoc 1993;270:2326-2330.

27. Tyson J, Malcolm S, Thomas PK, Harding AE. Deletions of chromosome 17p11.2 in multifocal neuropathies. Ann Neurol 1996;39:180-186.

28. Zuchner S, Mersiyanova IV, Muglia M, Bissar-Tadmouri N et al. Mutations in the mitochondrial GTPase mitofusin 2 cause Charcot-Marie-Tooth neuropathy type 2A. Nat Genet 2004;36:449-451.

29. Kijima K, Numakura C, Izumino H, Umetsu K et al. Mitochondrial GTPase mitofusin 2 mutation in Charcot-Marie-Tooth neuropathy type 2A. Hum Genet 2005; 116:23-27.

30. Reilly M. Axonal Charcot-Marie-Tooth disease: the fog is slowly lifting! Neurology 2005;65:186-187.

31. Lawson VH, Graham BV, Flanigan KM. Clinical and electrophysiologic features of CMT2A with mutations in the mitofusin 2 gene. Neurology 2005;65:197-204.

32. Khajavi M, Inoue K, Wiszniewski W, Ohyama T et al. Curcumin treatment abrogates endoplasmic reticulum retention and aggregation-induced apoptosis associated with neuropathy-causing myelin protein zero-truncating mutants. Am J Hum Genet 2005;77:841-850.

33. Hoogendijk JE, Hensels GW, Gabreels-Festen AA, Gabreels FJ et al. De-novo mutation in hereditary motor and sensory neuropathy type I. Lancet 1992;339:10811082.

34. Sereda MW, Meyer zu Hörste G, Suter U, Uzma N et al. Therapeutic administration of progesterone antagonist in a model of Charcot-Marie-Tooth disease (CMT-1A). Nat Med 2003;9:1533-1537.

35. Passage E, Norreel JC, Noack-Fraissignes P, Sanguedolce V et al. Ascorbic acid treatment corrects the phenotype of a mouse model of Charcot-Marie-Tooth disease. Nat Med 2004;10:396-401.

36. Nakamura Y, Shimizu H, Nishijima C, Ueno M et al. Delayed functional recovery by vincristine after sciatic nerve crush injury: a mouse model of vincristine neurotoxicity. Neurosci Lett 2001;304:5-8.

37. Naumann R, Mohm J, Reuner U, Kroschinsky F et al. Early recognition of hereditary motor and sensory neuropathy type 1 can avoid life-threatening vincristine neurotoxicity. Br J Haematol 2001;115:323-325. 